\title{
Caregiver and youth self-reported emotional and behavioural problems in Ugandan HIV-infected children and adolescents
}

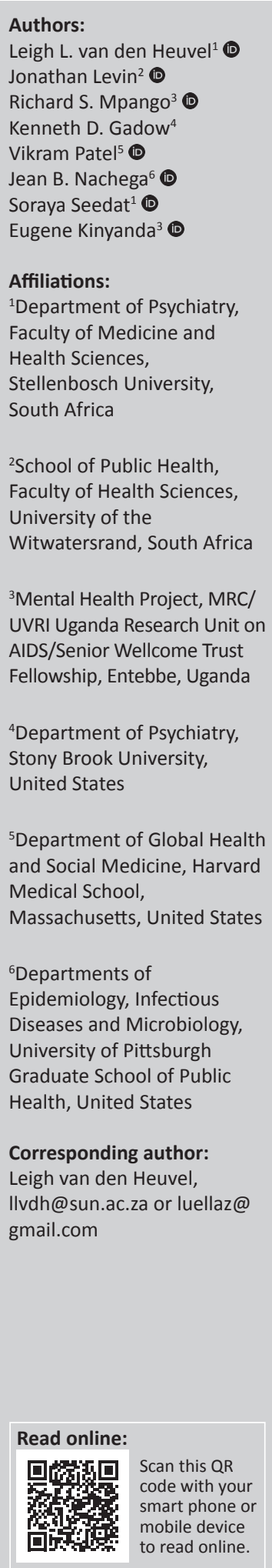

Authors:

Jonathan Levin ${ }^{2}$ (I)

Richard S. Mpango ${ }^{3}$

Kenneth D. Gadow

Vikram Patel ${ }^{5}$

ean B. Nachega ${ }^{6}$

Affiliations:

${ }^{1}$ Department of Psychiatry,

Stellenbosch University,

${ }^{2}$ School of Public Health,

Faculty of Health Sciences,

University of the

${ }^{3}$ Mental Health Project, MRC/ UVRI Uganda Research Unit on AIDS/Senior Wellcome Trust

${ }^{4}$ Department of Psychiatry, Stony Brook University,

United States

${ }^{5}$ Department of Global Health Medical School,

Massachusetts, United States University of Pittsburgh Graduate School of Public Health, United States

Corresponding author: Ilvdh@sun.ac.za or luellaz@ gmail.com
Introduction: We determined the prevalence of, and factors associated with, self-rated emotional and behavioural problems (EBPs) and assessed the agreement between self-rated and caregiverrated EBPs in the 'Mental health among HIV-infected Children and Adolescents (CA-HIV) in Kampala and Masaka, Uganda' (CHAKA) study. Existing literature demonstrates that CA-HIV face increased mental health challenges related to a broad range of biological and psychosocial factors. There is scarce data on self-reported EBPs in CA-HIV.

Methods: In a cross-sectional sample, caregiver-reported EBPs were assessed with the Child and Adolescent Symptom Inventory-5 (CASI-5), and self-reported problems were evaluated with the Youth Inventory-4 (YI-4) in 469 adolescents aged 12-17 years and the Child Inventory-4 (CI-4) in 493 children aged 8-11 years. Logistic regression models were utilised to determine factors related to self-reported EBPs.

Results: Self-reported emotional problems (EPs) were present in $28.8 \%$ of the adolescents and were associated with caregivers being separated and having a lower level of education. Among adolescents, $14.5 \%$ had self-reported behavioural problems (BPs), and these were associated with caregiver unemployment and food insecurity. Self-reported EPs were reported by $36.9 \%$ of children and were associated with rural study sites, having missed school and caregivers having a lower level of education. There was only modest agreement (maximum $r=0.29$ ) between caregiver- and CA-HIV-reported EBPS, with caregivers reporting more EPs and adolescents reporting more BPs.

Conclusion: Self-reported EBPs are frequently endorsed by CA-HIV, and these problems are related to unique psychosocial factors. Including CA-HIV, self-report measures can assist in identifying problems that caregivers may not be aware of, particularly BPs.

How to cite this article: Van den Heuvel LL, Levin J, Mpango RS, et al. Caregiver and youth self-reported emotional and behavioural problems in Ugandan HIV-infected children and adolescents. S Afr J Psychiat. 2018;24(0), a1265. https://doi.org/10.4102/sajpsychiatry. v24i0.1265

Note: A selected abstract from papers presented at the 19th National Congress of the South African Society of Psychiatrists in 'Professional Psychiatric Practice: Medical, Socio-Economic \& Cultural Perspectives', 21-24 September 2018, at the CSIR, Pretoria, South Africa. The congress is hosted by South African Society of Psychiatrists (SASOP).

Copyright: @ 2018 . The Authors. Licensee: AOSIS. This work is licensed under the Creative Commons Attribution License. 